\title{
Learning a causal structure: a Bayesian Random Graph approach*
}

\author{
Mauricio Gonzalez-Soto \\ Coordinación de Ciencias Computacionales \\ Instituto Nacional de Astrofisica Optica y Electronica (INAOE) \\ Mexico \\ mauricio@inaoep.mx \\ Ivan R. Feliciano-Avelino \\ Coordinación de Ciencias Computacionales \\ Instituto Nacional de Astrofisica Optica y Electronica (INAOE) \\ Mexico \\ ivan.feliciano@inaoep.mx \\ Luis E. Sucar \\ Coordinación de Ciencias Computacionales \\ Instituto Nacional de Astrofisica Optica y Electronica (INAOE) \\ Mexico \\ esucar@inaoep.mx \\ Hugo J. Escalante Balderas \\ Coordinación de Ciencias Computacionales \\ Instituto Nacional de Astrofisica Optica y Electronica (INAOE) \\ hugojair@inaoep.mx
}

\section{Introduction}

Random Graphs were proposed by Erdös and Renyi while using probabilistic methods in order to study problems in graph theory. A random graph can be thought of as a dynamic object which starts as a set of vertices and succesive edges are added at random according to some probability law. The simplest example consist of drawing at random a graph from the space of all graphs in $n$ vertices and $M$ edges, where each graph has the same probability (Bollobás. 2001). Further models can be found in complex systems, economics, the study of social networks among others (Jackson, 2010, Newman, 2018)

The concept of Causality deals with regularities found in a given environment (context) which are stronger than probabilistic (or associative) relations in the sense that a causal relation allows for evaluating a change in the consequence given a change in the cause. We adopt here the manipulationist interpretation of Causality (details in Woodward (2003)). The main paradigm is clearly expressed by Campbell and Cook (1979) as manipulation of a cause will result in a manipulation of the effect.

When doing Bayesian modelling (Bernardo and Smith, 2000; Gelman et al., 2013) one first identifies the source of the uncertainty; e.g., the parameter of a probability density function which generates data; then, one specifies a probabilistic model over such uncertainty. Here, we identify as our source

${ }^{*}$ https://arxiv.org/abs/2010.06164 
of uncertainty the existence or not of a causal relationship between a given pair of variables. We will model such uncertainty as the probability of an edge in a random graph. Our probabilistic model over the source of uncertainty is to be updated in terms of what is observed from interactions with the environment and therefore with the true causal mechanism that controls the environment.

\section{Methodology}

Let a rational agent consider the following set of variables $\mathcal{X}=\left\{X_{1}, \ldots, X_{n}\right\}$ which are causally related by some unknown, fixed causal graphical model $\mathcal{G}$; the agent knows that she can only intervene one variable, and does so in order to alter the value of some identified reward variable; without loss of generality assume that the agent can only intervene on $X_{1}$ wishing to affect $X_{n}$.

Also, we assume that the agent knows a causal ordering of the variables, which specifies, for some but not all of the variables, which other variables can not be a cause of it.

Let $p_{i j}$ be the belief that the agent has over a causal relation (directed link) existing between the variable with index $i$ and the variable with index $j$. This is, the decision maker has belief $p_{i j} \in[0,1]$ that $X_{i} \rightarrow X_{j}$. Let $G$ an initial random graph formed as follows: the node set is $N=\{1, \ldots, n\}$ and a there exists a link between $i$ and $j$ with probability $p_{i j}$. Now, make an intervention $a^{*}$ over the possible values that $X_{1}$ can take within the resulting graph $G$. The action is taken, and a full realization $X_{1}=x_{1}, \ldots, X_{n}=x_{n}$ is observed.

Next, we update the $p_{i j}$ 's using Bayes Theorem: for each pair of indexes $i, j$ we consider the subgraph containing only $1, i, j, n$ as nodes, either connected or not, and we ask for the probability of such graph producing the output $\left(X_{1}=a^{*}, X_{i}=x_{i}, X_{j}=x_{j}, X_{n}=x_{n}\right)$, which will be used as the likelihood of data, and as a prior probability we simply use $p_{i j}$, so we have

$$
p_{i j}^{t+1} \propto p\left(X_{1}=a^{*}, \ldots, X_{i}=x_{i}, \ldots, X_{j}=x_{j}, \ldots X_{n}=x_{n} \mid \text { current graph }\right) p_{i j}^{t} .
$$

Then, we update the model generating a new graph according to $p_{i j}$.

\section{Results}

We carried out a series of experiments in which an agent while acting on one variable at a time, updates its beliefs about the existence of a causal relationship between variables until they converge to a value that corresponds to whether the connection exists or not. Specifically, we examined the hypothetical example proposed by Gonzalez-Soto et al. (2018). Consider a patient who can have one of two possible diseases. A doctor can treat the disease with either treatment $A$ or treatment $B$, both of which carry some risk. Whether a patient is cured or not depends on the disease, the given treatment, and a possible negative reaction that the latter may have on the subject. We propose to mimic the physician-patient interaction with an agent interacting with an environment that is ruled by a causal model. Figure 1 shows the structure of the causal model.

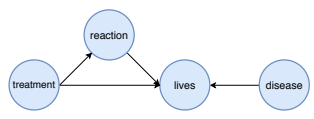

Figure 1: Causal structure underlying the disease-treatment problem.

We compare three algorithms each one has a different action selection policy. The first uses a random policy, and the two remain, use an $\epsilon$-greedy strategy starting with a high probability of explore and decaying the exploration rate until the agent only selects the optimal action. Figure 2 shows how the beliefs evolve when doing different interventions. In general, it is achieved what was expected, i.e. all the true relationships are learned. After a a few interventions, the system learns the causal model and at the same time learns in choose the best treatment. This gives a plus to other associative schemes.

To measure the performance of our algorithm we wish to know how different is the ground truth defined in Figure 1 and the beliefs. We use the $l^{2}$ norm, the Hamming distance, and the accuracy where we compare the values of the beliefs with the true edges. The first three plots of Figure 3 evidence that random actions are better to find the true causal relationships. On the other hand, we can see that policy using a fast decay of the exploration rate, outperforms the rest of the methods and 
is very similar to the Q-learning algorithm, a classical reinforcement learning method which is purely associative, with the same action selection scheme. However, our approach learns to choose from causal mechanisms of the world.

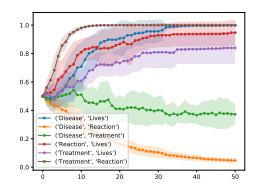

(a) Random policy.

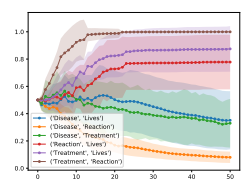

(b) $\epsilon \mathrm{w} /$ exponential decay.

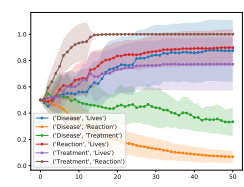

(c) $\epsilon \mathrm{w} /$ linear decay.

Figure 2: Average beliefs $p_{i j}$ over 50 rounds and 10 experiments.

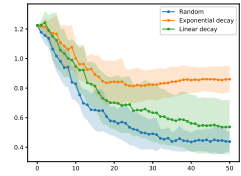

(a) $l_{2}$ loss

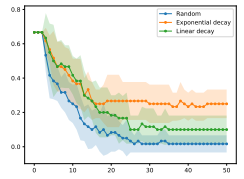

(b) Hamming loss

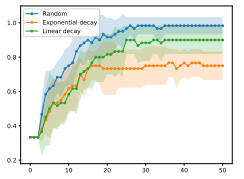

(c) Accuracy

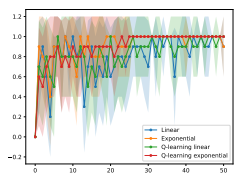

(d) Reward

Figure 3: Evaluation metrics per interaction round over 50 rounds and 10 experiments. 


\section{References}

Bernardo, J. M. and Smith, A. F. M. (2000). Bayesian theory. Wiley Series in Probability and Statistics.

Bollobás, B. (2001). Random Graphs. Cambridge studies in advanced mathematics.

Campbell, D. T. and Cook, T. D. (1979). Quasi-experimentation: Design \& analysis issues for field settings. Rand McNally College Publishing Company Chicago.

Gelman, A., Carlin, J. B., Stern, H. S., Dunson, D. B., Vehtari, A., and Rubin, D. B. (2013). Bayesian data analysis. CRC press, 3rd edition.

Gonzalez-Soto, M., Sucar, L. E., and Escalante, H. J. (2018). Playing against nature: causal discovery for decision making under uncertainty. In Machine Learning for Causal Inference, Counterfactual Prediction and Autonomous Action (CausalML) Workshop at ICML 2018.

Jackson, M. O. (2010). Social and economic networks. Princeton university press.

Newman, M. (2018). Networks. Oxford university press.

Woodward, J. (2003). Making things happen: A theory of causal explanation. Oxford Studies in Philosophy of Science. Oxford University Press. 